\title{
«Terranostra». I territori liberi delle alte valli del Taro e del Ceno. Estate 1944
} ISSN: 2282-4979 DOI: 10.12977/ereview102

Nell'estate del 1944 i comandi delle brigate partigiane che operavano nelle alte valli del Taro e del Ceno, nell'Appennino occidentale parmense, decisero di dare l'assalto ai presidi nemici ancora presenti nei principali centri delle due valli, dando così vita a due zone libere. Due esperienze di autogoverno che rivelarono però natura e specificità della Resistenza maturata in quella parte dell'Emilia, la sua complessità, le sue debolezze e lo slancio generoso che animava $i$ giovani combattenti anche al cospetto delle reali possibilità del movimento partigiano. Una esperienza di breve durata e forse non così importante nell'economia della lotta di liberazione in quella parte dell'Italia occupata, ma utile alla comprensione dello stato di sviluppo della Resistenza alla vigilia dell'arrivo del fronte di guerra lungo la linea Gotica.

In the summer of 1944 the headquarters of the partisan brigades operating in the Taro and Ceno valleys, in the Parmesan western Appennines, decided to assail the enemies' military posts, which were still present in the main villages of both the valleys. By doing so, they gave birth to two free zones - two experiences of local self-government, which revealed the nature and specificity of the Resistance movement in that part of EmiliaRomagna region. This means that, if on the one hand these experiences were animated by a generous enthusiasm of the young partisans, even when they were faced with the real possibilities of the Resistance, on the other hand they unveiled the complexity and weaknesses of the movement.

Those experiences were brief and maybe, in the end, not so highly important in the economy of Liberation strategy in that part of occupied Italy. Anyway, they can be useful for the comprehension of the Resistance developments at the dawn of the arrival of the war front along the Gothic line. 
All'inizio dell'estate del 1944 le alte valli del Taro e del Ceno, situate nell'Appennino occidentale Parmense al confine con la provincia di Piacenza, la Liguria e la Toscana, si trovavano ormai in larga parte sotto il controllo militare delle formazioni partigiane. Durante i primi sei mesi del 1944 «l'esercito partigiano» era cresciuto sia per numero di effettivi che per capacità militare. Superate le difficoltà iniziali - carenza di cibo, di armi e il costante pericolo di essere catturati - tutti ostacoli che sembravano dover limitare lo sviluppo della guerriglia sui monti dell'Appennino, i primi distaccamenti e gruppi di «ribelli» si trasformarono in pochi mesi in formazioni strutturate capaci di passare all'offensiva contro le postazioni nemiche. In alta val Ceno l'afflusso di uomini provenienti da Parma e dagli altri centri della valle, organizzati dal Partito comunista, che si aggiunsero ai giovani renitenti e disertori nativi di quei luoghi fecero del monte Barigazzo e dell'alta valle uno tra i maggiori centri di sviluppo della guerriglia in Emilia settentrionale. Territorio della 12a Brigata Garibaldi che aveva tra le sue fila molti giovani provenienti dal capoluogo e dalla pianura, «forestieri», portatori di mentalità, di abitudini e di idee politiche distanti da quelle che regolavano tradizionalmente la convivenza in quei luoghi. Giovani «ribelli» che chiedevano di essere ospitati e sfamati, e che facevano presagire possibili scenari di guerra e di violenza mai sperimentati prima in quelle terre, finendo per suscitare non solo diffidenza e fastidio tra le comunità locali ma anche paura e insicurezza. Una presenza che in alcuni settori della popolazione venne vissuta come una sorta di occupazione, creando i presupposti per una futura memoria antipartigiana che si sarebbe sedimentata nel tempo in una parte delle comunità a partire dall'indomani del 1945.

Per tutta la lotta di liberazione Bardi rappresentò comunque, per i garibaldini, una delle loro «piccole capitali». Fu nel villaggio di Osacca - Val Noveglia - a pochi chilometri dal centro dell'alta Val Ceno che si consumò il primo scontro a fuoco tra partigiani e fascisti, il giorno di Natale 1943, segnando l'avvio dello scontro armato e della guerra civile nel Parmense. È proprio a Bardi che reparti e combattenti colpiti da rastrellamenti e attacchi nemici si sarebbero riparati, anche dal Piacentino e dalle valli sul versante ligure; e da lì il comando della 12a Brigata Garibaldi (dalla quale sarebbero sorte tutte le altre che operarono nel Parmense) avrebbe condotto e organizzato le molteplici fasi della lotta fino alla liberazione definitiva.

Diversa la situazione in alta Val Taro dove protagonisti dell'attività di guerriglia furono i gruppi autonomi che si erano formati sul finire del 1943 e consolidati nella primavera del 1944, dando vita in estate alla 1a Brigata Julia, al Gruppo Centocroci, alla Brigata Beretta e ad alcuni altri distaccamenti e gruppi auto- 
nomi. Proprio per la forte presenza di combattenti nativi di quelle valli e per i loro legami con le comunità contadine l'insediamento della Resistenza si sarebbe rivelato assai più armonioso, favorito anche dal sostegno decisivo, sia nella fase di mobilitazione delle prime bande che nel sostegno per il prosieguo della lotta, da alcune figure autorevoli tra cui numerosi parroci. Inoltre va ricordato come le diverse formazioni partigiane autonome manifestarono la loro contrarietà ad una eccessiva politicizzazione della Resistenza e il loro fastidio verso le brigate Garibaldi, soprattutto quando penetravano nel loro territorio. Più inclini a interpretare la lotta di liberazione nei suoi aspetti militari e patriottici, i primi gruppi si erano infatti costituiti attorno ad alcune figure del posto, ex ufficiali e sotto-ufficiali, manifestando tiepidezza verso l'autorità espressa dal Cln provinciale.

Già da diversi mesi i distaccamenti garibaldini e i gruppi autonomi controllavano larga parte delle alte valli, mentre contingenti fascisti e tedeschi presidiavano i principali centri abitati, primi fra tutti Borgotaro, Bedonia e Bardi. La consapevolezza di avere mezzi e capacità per dare l'assalto ai presidi nemici non aveva fatto altro che alimentare il desiderio crescente tra i combattenti antifascisti di passare all'azione. Frenati fino a quel momento dai comandi superiori e dalle indicazioni dei centri politici clandestini di riferimento, consapevoli che una volta eliminati i presidi repubblicani e cacciati i reparti tedeschi le «terre liberate» andavano necessariamente difese, i partigiani non smisero di immaginare una azione corale per liberare le «loro» terre. Le perplessità di una parte dei comandi e dei dirigenti politici nascevano dalla consapevolezza che la liberazione avrebbe costretto le formazioni partigiane a forme di difesa statiche, snaturando di fatto una delle prerogative essenziali dell'azione di guerriglia che li aveva portati al successo in numerose azioni in quelle ultime settimane. Le formazioni partigiane si sarebbero trovate costrette a difendere un ampio settore del territorio appenninico senza

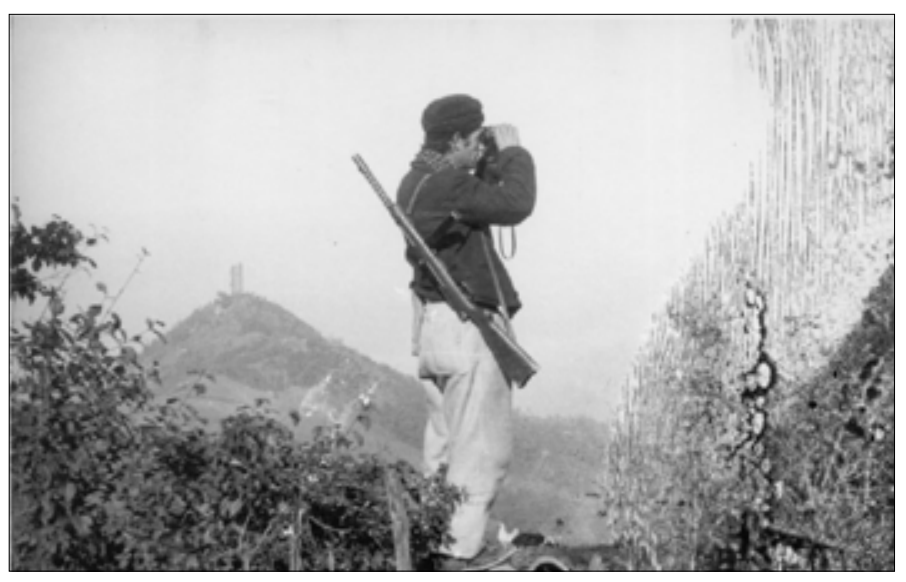

Vedetta partigiana al Passo del Bratello, Val di Taro averne la reale possibilità, esponendo le comunità contadine all'inevitabile reazione violenta dei fascisti e dei tedeschi. Ciò risultava particolarmente vero per l'alta $\mathrm{Val}$ Taro, vista la presenza di vie di comunicazione con- 
siderate vitali dall'esercito d'occupazione e con infrastrutture strategiche per i collegamenti tra la pianura e il fronte di guerra, prime fra tutte la stazione ferroviaria e la galleria del Borgallo a Borgotaro.

Dopo mesi trascorsi spostandosi tra boschi e pendii, lungo i monti che sovrastavano quelli che per una parte dei partigiani erano i paesi natii, per molti era giunto il momento di ritornare a casa da «liberatori». Sotto il profilo strettamente militare, i comandi si illudevano nell'ipotesi - sempre più convincente - che l'avanzata alleata avrebbe consentito alle armate anglo-americane di raggiungere il crinale appenninico prima dell'inverno. A quel punto gli eserciti alleati avrebbero trovato larga parte del versante emiliano dell'Appennino già controllato dai partigiani e la liberazione delle valli sarebbe diventata definitiva. Come sappiamo le cose andarono diversamente e invece dei liberatori a metà luglio sarebbero giunti i reparti tedeschi in rastrellamento.

Nelle settimane che precedettero la nascita dei «territori liberi», avvenuta nella seconda metà di giugno 1944, il numero degli effettivi e gli assetti militari della Resistenza subirono una robusta accelerazione, sospinta innanzitutto dal grande flusso di uomini che continuavano a raggiungere le alte valli in seguito alla chiusura del bando Graziani il 25 maggio e della liberazione di Roma il 4 giugno, ma anche dallo slancio impresso all'avanzata alleata verso nord. Alla fine di giugno in Val Ceno la 12a Brigata Garibaldi contava circa 500 partigiani, mentre le formazioni distribuite in Val Taro raggiungevano le 480 unità circa. Un migliaio di uomini animati dal desiderio di passare all'offensiva e avviare la liberazione a partire dalle loro valli.

Ad aumentare le differenze tra le esperienze che si stavano consumando nelle due vallate, l'arrivo in Val Taro all'inizio di giugno di Pietro Lavani "colonnello Lucidi", inviato dal comando militare di Milano con il compito di ispezionare le formazioni partigiane in alta valle e di valutarne l'efficienza. Accolto con interesse dai comandi partigiani delle diverse formazioni «autonome», il colonnello Lavani, probabile agente del Servizio informazioni militari del governo Badoglio e vicino a Raffaele Cadorna, mostrò fin da subito di essere lì con altri scopi. Andando oltre l'incarico ricevuto, nei numerosi incontri avuti con i gruppi partigiani sparsi nelle diverse località propose ai comandanti l'unificazione delle diverse formazioni della Val Taro in quella che doveva diventare, nei suoi programmi, la Divisione Nuova Italia, collegata politicamente con la Democrazia cristiana. L'iniziativa, in un primo tempo, sembrò avere successo: a Compiano - comune dell'alta valle - venne creato il Comando di Compiano, che avrebbe dovuto garantire una più equa distribuzione degli armamenti e una migliore organizzazione degli approvvigionamenti e finanziamenti alle diverse formazioni, fino ad arriva- 
re a costituire una cassa unica gestita dal Comando. Un progetto che certamente faceva progredire l'organizzazione militare delle formazioni in valle e li dotava di un riferimento politico in grado di garantire assistenza, rifornimenti e peso politico negli organismi collegiali della Resistenza. Non mancarono però le difficoltà. L'armonia all'interno del coordinamento non era sempre rispettata e i contrasti tra i diversi comandanti rese incerta la costituzione della Divisione, le cui sorti si sarebbero legate, a partire dalla metà di giugno, alle sorti del «territorio libero del Taro». Anche in questo il colonnello Lucidi ebbe un ruolo chiave nel determinare forma e conduzione della zona libera, riconducendola all'interno del suo progetto politico di unificare le formazioni partigiane operanti in alta valle sotto la tutela del Partito della democrazia cristiana.

Il progetto dovette anche fare i conti con il Comando di Milano. Ovviamente la sua attività non sarebbe passata inosservata ai comandi garibaldini e l'intervento del Comando del Corpo volontari della libertà $(\mathrm{Cvl})$ non avrebbe tardato ad arrivare, censurando l'operato del colonnello Lucidi per aver ampiamente travalicato i compiti ispettivi assegnatigli. Ciò che sarebbe accaduto poi è difficile da stabilire. A metà luglio il grande rastrellamento estivo travolse l'Appennino emiliano portandosi via le zone libere che avevano preso forma in quei mesi.

La completa liberazione dell'alta Val Taro, Bedonia e Borgotaro compresi, era forse già alla portata di mano delle brigate partigiane. I comandi erano però ben consapevoli che l'esercito d'occupazione non si sarebbe mai rassegnato alla perdita di controllo di strutture strategiche quali il tratto finale nel Parmense della linea ferroviaria Parma-La Spezia, la stazione ferroviaria di Borgotaro e la galleria, anch'essa ferroviaria, del Borgallo che collegava il capoluogo della val Taro con Pontremoli e La Spezia. Una pronta reazione con intervento militare per riprendere il controllo, almeno delle infrastrutture ferroviarie, non avrebbe tardato ad arrivare.

Apparentemente più semplice la conquista dei centri situati in alta Val Ceno. Per tutta la primavera le formazioni partigiane avevano eliminato progressivamente presidi e strutture nemiche e distrutto ponti e vie di collegamento, isolando il più possibile l'alta valle dai territori circostanti.

Nei primi giorni di giugno i preparativi per l'assalto ai capisaldi nemici subirono un'accelerazione, sostenuti dall'entusiasmo dei partigiani garibaldini ansiosi di iniziare quello che poteva rappresentare l'avvio della liberazione. Sottrarre completamente a tedeschi e fascisti l'alta valle serviva non solo a dare sbocco a mesi di impegno e di sacrifici, ma soprattutto a fornire all'esercito alleato in avanzata un trampolino di lancio verso la pianura padana e la completa liberazione del paese. 
Tutti comprendono che qualcosa di nuovo sta maturando - ricorderà uno di loro - Dario', con il suo gesto caratteristico, si frega le mani, solleva gli occhiali e dandosi una fregatina agli occhi esclama: «Ci siamo ragazzi. E' giunto il momento di uscire dalle nostre tane e scendere a valle per occupare i paesi» [Comitato per le celebrazioni del ventennale della Resistenza (ed.) 1965].

Il piano era semplice: attacco simultaneo dei centri della zona compresa tra Bardi e Fornovo Taro - a valle - con eliminazione dei presidi fascisti nei comuni di Bardi, Varsi e Varano dei Melegari e quello nella polveriera a Rubbiano. Prima di procedere, secondo le fonti garibaldine, Dario volle incontrare i comandanti delle brigate insediate nell'adiacente Val Taro - le brigate Julia, Beretta e Monte Penna - per verificare le condizioni per la liberazione simultanea delle due valli. L'incontro si tenne in località Caffaraccia (Borgotaro) e al termine di una articolata e sincera discussione, ricordano i testimoni, si concluse con un nulla di fatto. Non tutti i comandanti delle formazioni autonome erano convinti dell'utilità dell'azione. Dario e il resto del suo comando decisero di proseguire comunque con i piani prestabiliti.

Il 10 giugno 1944, alle ore 3 del mattino, i reparti partigiani attaccarono le postazioni fasciste in tutta l'alta valle. A fine mattinata i presidi repubblicani erano stati espugnati. L'alta Valle del Ceno era libera. La gioia di quei momenti sarebbe rimasta a lungo nella memoria di quanti combatterono quella battaglia. Così Luigi Sbodia, partigiano "Mario":

La battaglia si accende e divampa all'Ovest Cisa, ad uno ad uno cadono i presidi fascisti, verso l'imbrunire da Varano, Vianino, Varsi e Bardi si innalzano e dilagano per le valli i canti di vittoria che le pareti delle montagne rimandano in un'eco che sembra non voglia mai spegnersi: la Valle del Ceno è liberata [Sbodio 1964, $60]$.

L'eco del successo garibaldino in Val Ceno pose in allarme i presidi tedeschi e fascisti dislocati a Borgotaro e convinse gli scettici in Val Taro a passare anche lì all'attacco. La mattina del 15 giugno i primi reparti partigiani' entrarono festosi a Borgotaro. Il grosso delle truppe tedesche e fasciste nei giorni e nelle ore precedenti aveva nel frattempo abbandonato il capoluogo, sfruttando il passaggio nella galleria del Borgallo che collegava la stazione di Borgotaro con il versante pontremolese. Un «informatore» della Rsi rimasto in città descrisse l'ingresso 
dei partigiani in un suo rapporto al Duce. Esagerando per eccesso il numero di partigiani, egli stimò in «300 ribelli» l'avanguardia che prese la cittadina con «bandiere tricolore in testa e al canto di inni sovversivi», percorrendo «le vie della città applauditi dalle poche persone presenti e quindi prendeva possesso degli uffici pubblici e della stazione ferroviaria». Disarmati i militi della Gnr e della Guardia di Finanza rimasti in città, Borgotaro vene dichiarata città liberata. Nel corso della giornata altri reparti partigiani avrebbero fatto la loro comparsa a Borgotaro. Impressionò molto l'anonimo informatore l'arrivo, alle 11.30, di

[una] banda, della quale faceva parte certa 'Rosetta', professoressa di lettere di Borgotaro, vestita da uomo e armata di mitra [...]. Salutano, alcuni militarmente ed altri col pugno chiuso. Sono dotati di armi automatiche pesanti e leggere, di pistole mitragliatrici, pistole automatiche, bombe a mano e pugnali. Il munizionamento è abbondante. Vestono abiti civili di tela kaki e divise militari di panno grigio-verde. Intorno al copricapo portano un nastro rosso; sulla visiera una stella a cinque punte od una coccarda tricolore. Indumenti e calzature sono, in genere, in buono stato. Quasi ogni notte aerei nemici riforniscono la banda di generi alimentari, armi, munizioni e indumenti. Sembra che la 'Brigata Julia', che ha fatto saltare vari tratti della linea ferroviaria Parma-La Spezia, abbia intenzione di occupare militarmente tutti i centri abitati della Valle del Taro ${ }^{3}$.

Nel corso della giornata che aveva visto i patrioti accedere all'abitato di Borgotaro fecero la loro comparsa in centro città inaspettatamente due autovetture tedesche, arrivate dal passo Centocroci ignare dell'avvenuta liberazione. Seguirono momenti di tensione seguiti da una intensa sparatoria mentre i militari cercavano di uscire dall'abitato. Esito dello scontro: un partigiano morto, i tedeschi fatti prigionieri e acquisizione da parte dei resistenti di una ricca documentazione militare da inviare al comando generale militare Cvl a Milano. L'incidente convinse i comandi partigiani, se ve ne fosse stato bisogno, delle difficoltà di difesa di Borgotaro e della necessità di spostare gli uomini all'esterno del perimetro urbano, sulle alture circostanti a difesa del borgo. Il giorno seguente il maggiore Koeppers informava telefonicamente il maggiore von Keller che tutti i partigiani si erano ritirati dal paese: «Quando la sera del 16 giugno il gruppo Almers entrò a Ostia e Borgo Val di Taro, non trovò più nessun partigiano. Anche i civili erano fuggiti sui monti non appena avevano saputo dell'arrivo delle unità tedesche. In seguito il fianco settentrionale del Monte Molinatico, dove si erano ritirati i 
partigiani, venne rastrellato dopo che la parte meridionale era stata sbarrata» ${ }^{4}$. A questo punto le fonti si fanno meno chiare: non si hanno notizie precise sulla presenza dei distaccamenti nel centro abitato così come sulla nascita dell'autogoverno partigiano, almeno fino al 26 giugno quando la presenza antifascista tornò a essere consistente nel borgo.

La liberazione di Borgotaro, Bedonia e degli altri centri abitati dell'alta valle alimentò anche qui l'entusiasmo tra i partigiani della valle, come scriverà il partigiano "Italo":

[un'] euforia generale che mette le ali alla fiducia e alla speranza e relega nel subcosciente il timore e il dubbio d'un responso altrimenti negativo. L'avere conteso e strappato questo territorio in termini fino a poco prima inimmaginabili alle forze locali di quello che è il più potente e temuto esercito del mondo infondono ai nostri uomini nuovo slancio e fiducia [Lodi 1985, 46].

Tracciati i confini, inevitabilmente labili, che includevano i territori di Albareto, Bedonia, Compiano, Tornolo e ovviamente Borgotaro - circa 2.500 chilometri quadrati, sempre secondo il partigiano Italo componente del comando della 1a Brigata Julia - per la Resistenza divenne indispensabile difenderli, impegno assai dispendioso vista la volontà tedesca di riconquistare quanto prima il controllo di Borgotaro e delle infrastrutture ferroviarie. Accanto, in Val Ceno, i confini della zona libera compresero i comuni lungo l'asse nord-sud del fiume: da Varano De' Melegari sino a comprendere Varsi e Bardi. Anche qui le minacce potenziali provenivano dai territori confinanti: dalla Liguria, dal Piacentino e dai centri lungo la pedemontana, Salsomaggiore Terme soprattutto. A difesa della valle oltre ai reparti partigiani, l'impraticabilità delle vie di comunicazioni e in ultima istanza la convinzione che ai tedeschi quel territorio non interessasse granché e che la sua conquista non fosse al centro dei loro programmi militari.

$\mathrm{Al}$ rientro da Bardi, dove si era recato in autoambulanza per effettuare uno scambio di prigionierí, l'ufficiale medico Rüll fece rapporto ai propri superiori. Ne emerge una descrizione preziosa del territorio e delle persone che incontrò durante il suo viaggio, tutt'altro che semplice per lui, ma pieno di sorprese.

Superammo Fornovo e, poiché poco prima, dopo Varano, il ponte era saltato, fummo costretti ad attraversare il fiume ad un guado, cosa che fu possibile solo con il traino di due buoi e con grandi difficoltà con un'interruzione di un'ora e mezza. 
Già qui l'intera zona si era rivelata dominata dalle bande. I giovani salutavano con il pugno alzato, in parte erano armati con mitra, ma ci aiutarono molto volentieri nella manovra per disincagliare l'ambulanza. Oltre il ponte proseguimmo su una strada in buono stato. Il comandante dei banditi aveva preso posto sul tetto del mezzo con un tricolore per garantirci di transitare liberamente. Tutti i passanti, non molti, e gli abitanti delle fattorie lungo la strada lo salutarono con il pugno alzato. Le persone impegnate nei campi per il raccolto ci ignorarono.

Prima della località di Vianino dovemmo lasciare l'autoambulanza perché anche lì il ponte era distrutto e non vi erano deviazioni possibili. Era questo il punto stabilito per lo scambio dei prigionieri. Lasciai il sotto-ufficiale tedesco di sanità e i tre soldati italiani con i prigionieri da scambiare, presi una barella e qualche strumento medico. Dovetti lasciare anche la pistola [...]. Attraversammo in tre il letto del fiume quasi asciutto e il pendio molto ripido di fronte, il capobanda, il poliziotto ferito e io. Le alture circostanti erano visibilmente occupate dai banditi. Molti ci vennero incontro, tutti giovani robusti, tra i 18 e i 22 anni, tutti vestiti da civili con pantaloni corti, camicie con le maniche rimboccate, polpacci scoperti e fazzoletto rosso al collo. Alcuni portavano cappelli di feltro verde e rosso con nastro con i colori italiani e stelle a cinque punte. Portavano stelle a cinque punte, bianche rosse e verdi ricamate, anche sulle camicie. Durante l'intera ascesa feci attenzione a fortificazioni e postazioni difensive, ma non vidi nulla [...].

Proseguimmo in auto per Bardi che raggiungemmo presto. La località sorge in modo molto pittoresco su un'altura circondata dalle montagne. Caratteristico è un vecchio castello che si scorge in lontananza. Ci sono molte ville abitate dalla plutocrazia italiana che ancora oggi gode il fresco estivo indisturbata dalla guerra e dalle bande. L'atteggiamento di simpatia verso le bande le garantisce questa esistenza indisturbata. Incontrai per poco il comandante in capo dei banditi, un giovane di circa 25 anni, di aspetto semita. Mi sembrò piuttosto insicuro nei modi e nel comportamento. Il quartier generale si trova all'ingresso del paese, in un grande edificio nuovo che prima, probabilmente, era la Casa del Fascio.

Le formazioni partigiane che ora potevano godere di una relativa sicurezza interna, si dovettero però far carico delle condizioni degli abitanti delle zone libere e delle insidie che provenivano dall'esterno. Poterono rafforzare la propria capacità militare, ricevere rifornimenti tramite i lanci aerei dagli alleati, accogliere e armare il gran numero di renitenti e disertori che giungevano sui monti disarmati e poco avvezzi alla guerriglia, misurarsi con la politica attraverso 1'istituzione di amministrazioni libere.

In Val Ceno, nei giorni successivi alla liberazione di Bardi, la popolazione venne convocata nella piazza del paese - non sappiamo se i soli capifamiglia o tutta 


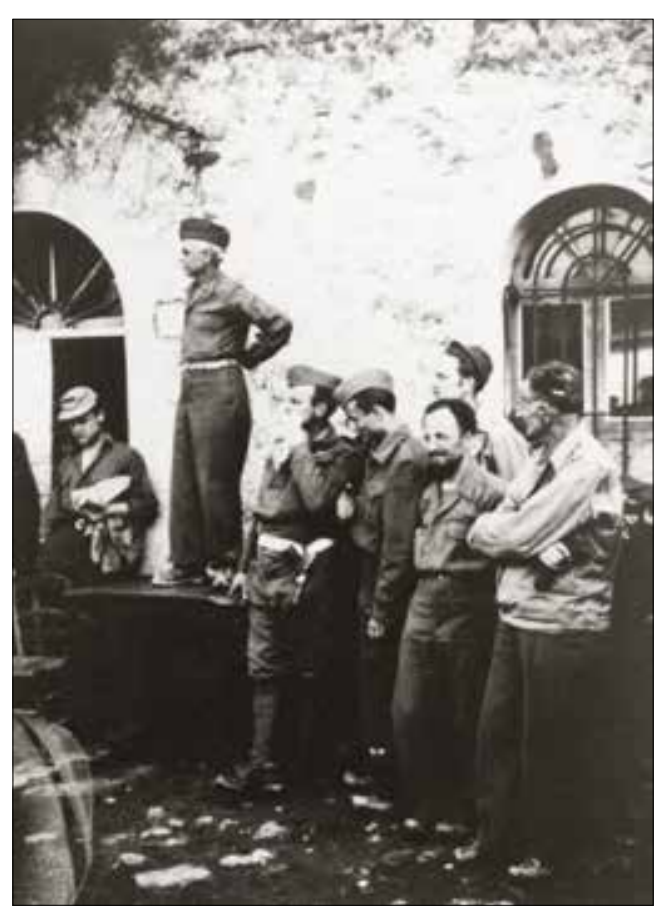

Il Prefetto Achille Pellizzari tiene un discorso ai partigiani in alta val Taro la popolazione comprese le donne - e le venne proposta l'elezione dell'avvocato Giuseppe Lumia a sindaco. Così, a guerra conclusa, avrebbe ricordato l'evento lo stesso Lumia:

Il Comandante della Brigata prese per primo la parola per rivolgere un cordiale ringraziamento alla popolazione per l'affettuosa, paterna assistenza di cui aveva circondato $\mathrm{i}$ Patrioti, illustrò poscia i motivi ideali che indussero i giovani a pigliare le armi contro il malgoverno fascista e, infine, interpellò il Popolo se voleva proclamare, come Sindaco, l'avv. Giuseppe Lumia. La folla, a gran voce, rispose affermando [G. Lumia 1945, 21].

A Borgotaro le cose andarono diversamente e non solo per le difficoltà nell'organizzare la difesa

del paese dalla minaccia tedesca. Ci vollero alcune settimane prima che i comandi partigiani riuniti alla presenza del colonnello Lucidi si occupassero dell'«amministrazione civile della zona liberata». Scelsero di applicare il modello statale nominando un prefetto con funzioni esecutive che avrebbe, a sua volta, promosso la nascita di organismi amministrativi. La scelta ricadde su Achille Pellizzari, 61 anni, docente di letteratura italiana presso l'Università di Genova, già deputato del Partito popolare negli anni 1921-23 e schedato come sovversivo [Mastrodonato 2015; Franchino 1976; Pellizzari 1978]. Avvertito della decisione presa mentre si trovava nascosto poco lontano, nel vicino comune di Berceto, venne condotto a Compiano presso il comando della costituenda Divisione La Nuova Italia e nominato prefetto del «territorio libero del Taro» il 3 luglio 1944. Tra i suoi prima atti la nomina a sindaco di Bedonia di Mario Serpagli e la costituzione della Giunta democratica di Borgotaro composta da notabili locali e cittadini meritevoli di fiducia secondo i comandi partigiani ${ }^{7}$. Al fine di celebrare 
la nascita della zona libera venne pubblicato il foglio "La Nuova Italia", stampato presso la locale tipografia Cavanna.

In entrambe le zone libere, malgrado la buona volontà, gestire l'amministrazione civile risultò assai complicato, principalmente per la scarsità di risorse disponibili e la brevità dell'esperienza politica. Nelle poche settimane in cui poterono operare le due amministrazioni - un mese circa quella di Bardi e una ventina di giorni circa quelle di Borgotaro e Bedonia - l'impegno maggiore profuso consistette nell'organizzare la distribuzione del grano e dei prodotti agricoli nel tentativo di ridurre, se non addirittura abolire, l'aggravio fiscale a carico delle famiglie e del poco commercio ancora esistente.

In Val Taro, in particolare, ricorda Giacomo Vietti nel suo L'Alta val Taro nella Resistenza:

si organizza la distribuzione dei viveri e si istituiscono tribunali militari per l'amministrazione della Giustizia, sia in materia penale [ch]e civile, si pubblicano bandi per reprimere il contrabbando dei generi alimentari e viene istituito un corpo di polizia partigiana che continuerà a svolgere funzioni amministrative anche dopo l'occupazione tedesca. Si distribuisce ai familiari dei patrioti parte della legna prelevata dai depositi dei fascisti e che era in attesa di essere trasportata a Parma. Una precisa contabilità tenuta dal Comando di Polizia di brigata permette di quantificare la qualità di legna e carbone distribuito gratuitamente alle famiglie partigiane, ed alle famiglie bisognose e la quantità di combustibile venduto: quintali $4.987,8$ di legna, quintali 123,2 di carbone di legna. Incasso L. 120.000 .

Si controllano i lavori di trebbiatura in modo da prelevare in modo equo i contingenti di grano, sia per le formazioni partigiane che per la popolazione civile. Anzi a Borgotaro sarà la polizia partigiana che riuscirà $\mathrm{a}$ procurarsi il carburante e l'olio lubrificante per il funzionamento delle trebbiatrici, materiali forniti dalla Fabbrica del Cemento che ne aveva una cospicua scorta. Si lavorava per ripristinare il campo d'aviazione nella previsione di poterlo utilizzare per il collegamento aereo con gli

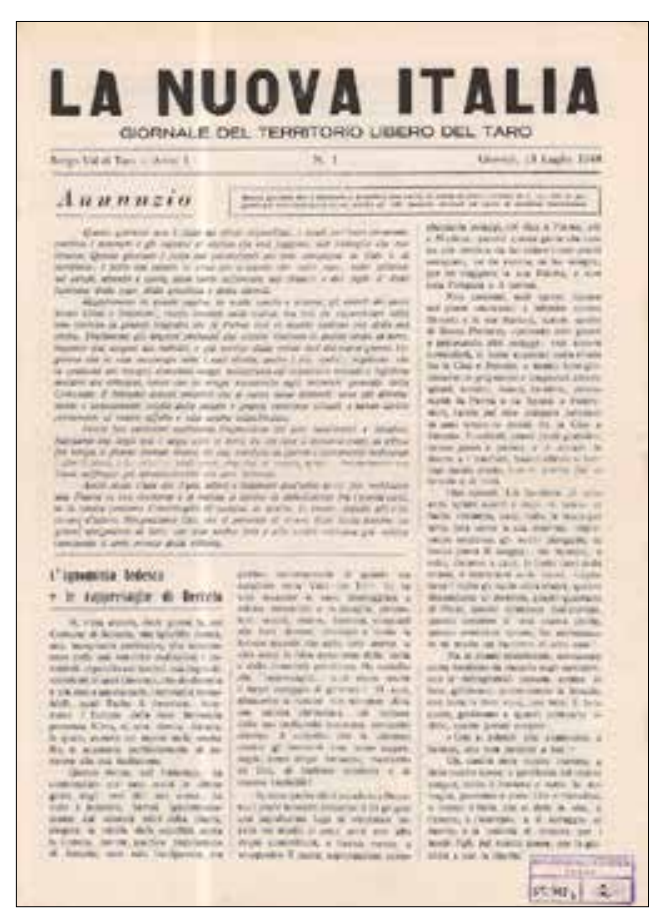

Giornale del Territorio libero del Taro 
alleati dei quali ci si attende un imminente sbarco in Liguria [Vietti 1980, 201]. A Bardi, il sindaco Lumia tentò la messa in atto di un piano per il recupero di risorse economiche per finanziare progetti in favore della popolazione. Interrotti i rapporti con la Prefettura di Parma, chiusa la filiale della Cassa di Risparmio, abolito il dazio consumo, abrogate le imposte sindacali - considerate «norme fasciste» - e decurtate le altre, come voluto dai comandi partigiani, l'«amministrazione civica popolare» si ritrovò priva di mezzi finanziari per sviluppare un qualsivoglia programma di interventi in favore della comunità. Lumia convocò «una trentina dei più abbienti» del territorio, ma «la riunione non diede risultati di sorta». Il sindaco non si diede per vinto e alla fine riuscì a convincerli a concedere un prestito, assumendo personalmente l'impegno dell'integrale restituzione della somma ai creditori. Il progetto prevedeva che l'amministrazione emettesse titoli quando se ne fosse presentato il bisogno e finanziasse così il debito contratto con il gruppo di benestanti che avevano accettato di prestare le risorse all'ente di governo locale. Naturalmente il piano prevedeva, o per lo meno si augurava, che al «territorio libero di Bardi» sarebbe seguita la liberazione definitiva del paese con l'integrazione della zona libera nell'Italia liberata. Molti, infatti, contavano sull'arrivo degli Alleati entro l'inverno. Al posto dei liberatori giunsero invece i rastrellamenti dell'Operazione Wallenstein, che cancellarono ogni esperimento partigiano di controllo diretto del territorio in Emilia.

L'impegno maggiore per la Resistenza anche durante queste settimane rimase comunque la difesa militare dei confini delle zone libere. Particolarmente in Val Taro le brigate partigiane, insidiate da reparti nemici provenienti da diverse direttrici, difesero con astuzia e coraggio le zone liberate. Attacchi vennero portati nella valle del Manubiola, affluente del torrente Taro, e in località Grifola, località a sud ovest del capoluogo, puntualmente respinti al termine di duri scontri a fuoco con vittime da ambo le parti. Una difesa accanita quella messa in atto dai reparti partigiani, che si trovarono a dover fronteggiare anche incursioni aeree alleate. Male informati - l'aviazione non era infatti al corrente della liberazione del capoluogo - gli anglo-americani colpirono più volte le infrastrutture ferroviarie e l'ospedale, provocando morti e feriti tra i reparti partigiani e la popolazione civile. Mentre a poca distanza, nei medesimi giorni, gli stessi Alleati effettuarono diversi lanci in favore dei partigiani della valle.

Nella valle accanto la situazione si presentava differente. Assai meno rilevante per le infrastrutture che l'attraversavano, aveva però fama di essere uno dei maggiori centri della Resistenza nella vasta area compresa tra le Valli del Taro e del Trebbia, a cavallo tra le province di Parma e Piacenza e confinante con la Liguria. Da lì partivano i reparti partigiani che portavano gli attacchi ai convogli 
tedeschi in transito sulla Via Emilia, che tanto danneggiavano l'esercito d'occupazione. Gruppi di guerriglieri scendevano a valle al tramonto percorrendo decine di chilometri per raggiungere la statale di notte e compiere le loro azioni. L'intraprendenza partigiana in quel settore rappresentava per i comandi tedeschi un serio pericolo per la circolazione di mezzi e uomini lungo la Via Emilia, arteria di collegamento nel retro fronte di vitale importanza, ma che poteva diventare elemento di pericolosità in caso di sbarco alleato in Liguria. La proclamazione di territorio libero non fece altro che conferire a Borgotaro lo status di piccola capitale partigiana, rendendola come tale un obiettivo da colpire.

Il 18 luglio 1944 reparti della Luftwaffe, del $12^{\circ}$ Reggimento di Polizia, delle SS, del SD, della Feldgendarmerie affiancati dal Battaglione Lupo della X Mas invasero la Valle del Taro: era iniziata l'Operazione Wallenstein II. La seconda delle tre fasi del grande rastrellamento nazista che colpì l'Appennino emiliano, nei confronti del quale nulla poterono le difese partigiane, costrette ad abbandonare i territori liberati mentre le truppe in rastrellamento si accanivano contro le comunità contadine, vittime di saccheggi, deportazione e stragi. Una lunga scia di sangue annullò - seppur solo temporaneamente - la presenza partigiana in quei luoghi, cancellando l'esperienza delle zone libere del Taro e del Ceno. Con la fine del «territorio libero del Taro» si interruppe anche il processo di costituzione della Divisione Nuova Italia in seguito alla morte del suo ideatore, il colonello Pietro Lavani, ucciso dai tedeschi al passo dei Due Santi insieme al suo aiutante Macchi durante il rastrellamento.

Concluse le operazioni della Wallenstein II, la terza fase del rastrellamento venne orientata verso il Modenese contro la «Repubblica partigiana di Montefiorino», e il ritiro delle truppe dal Parmense riportò le brigate partigiane a reinsediarsi nelle valli e lungo i pendii dei monti che li avevano visti protagonisti durante la prima metà del 1944. In nessuna delle valli del Parmense sarebbero risorti «territori liberi», sebbene si riproponesse il problema della gestione e della difesa delle aree d'insediamento partigiano. Un aspetto della lotta di liberazione che avrebbe accompagnato i comandi partigiani fino alla sconfitta militare dei nazi-fascisti.

Nelle settimane e mesi che seguirono la fine del rastrellamento Bardi e larga parte dell'alta Val Ceno tornarono ad essere territorio partigiano, subendo altri rastrellamenti che provocarono ulteriori morti, feriti e deportati. Su Borgotaro invece continuò la pressione dell'esercito tedesco, deciso a mantenere il controllo delle infrastrutture ferroviarie, della stazione e della galleria del Borgallo. I partigiani, dal canto loro, consapevoli di non essere in grado di reggere lo scontro e di non avere i mezzi militari per sopportare altri pesanti rastrellamenti, altre stragi e altre distruzioni come quelle subite durante le ultime settimane di luglio, scelsero altre 
soluzioni di natura militare. Va anche ricordato che larga parte dei partigiani delle brigate presenti in Val Taro avevano in quegli stessi territori casa e famiglia, che ovviamente intendevano liberare, ma contemporaneamente anche salvaguardare dalla politica stragista dell'esercito tedesco.

Da qui la decisione da parte dei comandi delle brigate che operavano in Val Taro di scendere a patti col nemico e acconsentire la stipula di accordi con i comandi tedeschi sulla gestione del territorio. Il 22 luglio 1944 - a pochi giorni di distanza dalla fine delle operazioni di rastrellamento - lo stato maggiore della 2a Luftflotte, che aveva condotto le operazioni di rastrellamento, annunciava l'avvio delle trattative con i comandi partigiani della valle. Accordo che sembrò dover naufragare per la condizione posta dai partigiani determinati a far ritirare il Battaglione Lupo della X Mas dalla valle, provocando inutili proteste del generale Graziani. Superate le difficoltà venne stipulata una tregua, che non sembra sia stata di breve durata come spesso accadde in questi casi ${ }^{8}$. L'accordo - come scrive Carlo Gentile - a metà settembre era infatti ancora in vigore, come osserva l'SS-Obersturmbannführer Werner Grothmann, aiutante di Himmler, in un rapporto indirizzato all'ufficiale Ia della 16a SS-Panzer-Grenadier-Division Reichsführer-SS Ekkehard Albert: «Per quanto concerne il capobanda Beretta, dovrete attenervi anche voi agli accordi in vigore. Ammetto che la cosa lascia perplesso anche me, ma la decisione è stata presa 'in alto'» [Gentile 2015, 166-167].

\section{Bibliografia}

Comitato per le celebrazioni del ventennale della Resistenza (ed.) 1965, Celebrazione del ventennale della liberazione della Val Ceno. 1944-1965, Parma: Step

F. Franchini 1976, Achille Pellizzari, Partigiano Poe. Raccolta di documenti e testi, La Spezia: Fivl

C. Gentile 2015, I crimini di guerra tedeschi in Italia, Torino: Einaudi

L. Klinkhammer 1993, L'occupazione tedesca in Italia, 1943-1945, Torino: Bollati Boringhieri

M. Lodi 1985, Obiettivo libertà. Storia della «la Julia». Brigata partigiana dell'alta Val Taro, Parma: Alpi

G. Lumia 1945, Bardi centrale di patriotteria, Parma: Tip. Fratelli Godi

A. Mastrodonato (ed.) 2015, Vite ritrovate. Achille Pellizzari. Un educatore nella Resistenza, Parma: Isrec Parma 
R. Mira 2011, Tregue d'armi. Strategie e pratiche della guerra in Italia fra nazisti, fascisti e partigiani, Carocci: Roma

P. Pellizzari 1978, Storia della più piccola capitale del mondo, Compiano: Arte Storia

L. Sbodio 1964, Fornovo Taro nel movimento partigiano. Cronistoria, II edizione, Parma: Step

L.Tarantini 1978, La Resistenza armata nel Parmense, Anpi: Parma

G. Vietti 1980, L'Alta Val Taro nella Resistenza, Parma: Anpi

Centro studi movimenti (ed.) 2015, Una stagione di fuoco. Fascismo, guerra, Resistenza nel Parmense, Parma: Fedelo’s “La Nuova Italia”, n. 1, 13 luglio 1944 